\title{
Objective-type dark-field illumination for scattering from microbeads
}

\author{
Ido Braslavsky, Roee Amit, B. M. Jaffar Ali, Opher Gileadi, Amos Oppenheim, and \\ Joel Stavans
}

\begin{abstract}
We introduce a method for detecting and tracking small particles in a solution near a surface. The method is based on blocking the backreflected illumination beam in an objective-type total internal reflection microscope, leaving unhindered the light scattered by the particles and resulting in dark-field illumination. Using this method, we tracked the motion of $60-\mathrm{nm}$ polystyrene beads with a signal-tonoise ratio of 6 and detected 20 -nm gold particles with a signal-to-noise ratio of 5 . We illustrate the method's use by following the Brownian motion of small beads attached by short DNA tethers to a substrate. (C) 2001 Optical Society of America
\end{abstract}

OCIS codes: $110.0180,120.1880,120.5820,290.5850$.

\section{Introduction}

The detection of the motion of particles near a surface is a powerful method for monitoring a variety of physical, chemical, and biological processes. Examples include measurements of colloidal forces, ${ }^{1}$ DNA elasticity, ${ }^{2}$ DNA-protein interactions ${ }^{3-5}$ at the singlemolecule level, and the unfolding of proteins. ${ }^{6} \mathrm{~A}$ common feature of these studies is the large size of the particles used, which ranged between 2 and 20 $\mu \mathrm{m}$. Within this size range, particles such as polystyrene beads scatter light strongly, and therefore their motion can be detected easily with simple devices such as photodiodes.

In many applications, particularly in biology, one is interested in tracking the motion of much smaller particles attached to a molecule of interest. An example is the recent study of protein-mediated DNA

When this research was performed, I. Braslavsky, R. Amit, B. M. Jaffar Ali, and J. Stavans (joel.stavans@weizmann.ac.il) were with the Department of Physics of Complex Systems and O. Gileadi was with the Department of Molecular Genetics, Weizmann Institute of Science, Rehovot 76100, Israel. A. Oppenheim was with The Hebrew Universtity, Hadassah Medical School, P.O. Box 1172, Jerusalem 91010, Israel. The current address for I. Braslavsky is the Department of Applied Physics, Caltech, Pasadena, California 91125. The current address for B. M. Jaffar Ali is the National Center for Biological Sciences, Tata Institute of Fundamental Research, GKVK Campus Bangalore 560012, India.

Received 22 January 2001; revised manuscript received 6 July 2001.

0003-6935/01/315650-08\$15.00/0

(C) 2001 Optical Society of America looping, ${ }^{7}$ in which changes in the Brownian motion of $0.22-\mu \mathrm{m}$ polystyrene beads tethered to a surface by short DNA molecules were followed. The need to use small particles stems from the requirement that particles interfere as little as possible with the phenomenon under study. One problem posed by the use of small particles is their detection: The scattering cross section decreases as the sixth power of the bead size in the Rayleigh-Gans approximation. ${ }^{8}$ This precludes the detection of small beads with bright-field illumination. More sophisticated schemes such as fluorescence detection, or differential interference contrast (DIC), which allows the observation of particles of tens of nanometers, are then necessary.

In this paper we demonstrate a fluorescenceless method for detecting particles tens of nanometers in size. The method, an alternative to DIC, involves simple modifications to an objective-type total internal reflection microscope (TIRM) similar to that developed by Stout and Axelrod. ${ }^{9}$ These authors discussed in detail the advantages of an objective to create evanescent illumination, as opposed to the traditional method in which a prism is employed. An objective-type TIRM microscope takes advantage of the large angles of incidence of rays produced by illuminating the back aperture of a large numerical aperture (NA) objective either with annular illumination or with off-axis rays. A drawback of this arrangement is that rays that are totally reflected by the glass-water interface can enter back into the objective. Without use of a filter as in fluorescence applications, these rays may wash out the scattering 
signal of a small bead. We therefore blocked these rays by inserting a field stop at an appropriate location along the optical path. This resulted in darkfield illumination that allowed us to detect with a high signal-to-background ratio polystyrene beads as small as $60 \mathrm{~nm}$ and gold particles $20 \mathrm{~nm}$ in diameter with a simple CCD camera. Since we detect the light scattered by objects in the sample, we call the method objective-type dark-field scattering microscopy (ODFSM). We point out that various obliqueray illumination schemes and beam blocks have been used in the past to improve image contrast.10,11 ODFSM constitutes an improvement over these schemes.

In Section 2 we present two different schemes for implementing ODFSM and also describe in detail the necessary modifications for achieving ODSFM conditions in a commercial microscope. Use of the technique is illustrated by measurement of the Brownian motion of small beads tethered to a glass slide by short DNA molecules of different lengths.

\section{Materials and Methods}

In their setup Stout and Axelrod illuminated the back aperture of a large NA objective with a beam of annular cross section, produced by blocking the central portion of an expanded laser beam with an opaque disk. This latter was placed along the optical path at a plane conjugate to the back focal plane (BFP) of the objective, the equivalent back focal plane (EBFP). The diameter of the disk was chosen so as to allow only those rays through that are totally reflected at the glass-water interface. These rays entered back into the objective but were rejected by an emission filter that allowed the fluorescent signal through.

In ODFSM we also take advantage of a high NA objective and annular illumination to detect light scattered by the particles of interest. The scattered light is of the same wavelength as the light reflected back by the glass-water interface into the objective. Since the intensity of the latter is much higher, it washes out the scattered light we want to detect. To eliminate the reflected rays, we placed a field stop at a second EBFP formed on the return path of the image by an imaging lens in such a way as to let through the light scattered by the particles alone. Note that in contrast with the objective-type TIRM, annular illumination is primarily used in ODFSM to eliminate reflected rays with ease and not necessarily to produce evanescent illumination.

We illustrate these ideas in Figs. 1 and 2 where we show two possible ways to implement our method around an inverted microscope. In Fig. 1 a diffractive element made of a binary surface with a radial phase grating is used to convert the laser beam cross section into a thin expanding annulus. ${ }^{12}$ Lens L1a forms an image of the annulus at its focal plane. This annulus is imaged on the back focal plane of the objective, BFP, by a second lens, L2a. Therefore the focal plane of lens L1a is an equivalent back focal plane, EBFP1. Residual traces of zeroth-order illumination from the diffractive element are eliminated by an opaque disk at this plane, whose diameter is smaller than the annulus's inner diameter. The relative distances between the four optical components, L1a, L2a, the diffractive element, and the objective, are set so that the outer diameter of the annular illumination impinging on the BFP of the objective is of the same size or slightly larger than the objective's back aperture. The focus of lens L2a is conjugated to the image plane because the sample is located at the focus of the infinity-corrected objective. To get nonannular illumination at the sample plane, the distance between the diffractive element and L1a is adjusted so that a spot (an image of the beam impinging on the diffractive element) is formed at the focus of L2a. We emphasize that the distance between the diffractive element and L1a does not change the size or the location of the annulus at the focus of L1a. However, it changes both the location and size of the image of the impinging beam at the diffractive element plane. This feature is used in the aligning process of the system as explained above. Proper beam steering into the objective is achieved by means of a beam splitter, BS. To block the rays reflected at the glass-water interface, part of which is transmitted through the beam splitter, a field stop is placed at a plane, EBFP2, conjugate to the BFP, formed by lens L3a. This field stop, a hole in a screen, blocks the annular-shaped image of the rays emerging from the objective, creating a dark-field effect. Finally, lens L4a is used to form an image of the scattering objects on the CCD. The need for L4a arises from the fact that the location of EBFP2 lies beyond the focal plane of L3a, in which an image of the sample plane is formed. Note that if the diameter and width of the annular illumination are chosen appropriately, total internal reflection conditions can be attained. We point out, however, that pure evanescent illumination is hard to achieve by objective-type methods (see Section 4).

Note that in this configuration a spot of limited size on the sample is illuminated. The size of the illumination spot, of $\sim 30 \mu \mathrm{m}$ in our case, is proportional to the cross section of the laser beam as it impinges on the diffraction element. This is because the cross section determines the size of the spot at the focus of lens L2a, since the location of the diffraction element and the focus of L2a are conjugated by L1a. We illustrate this in Fig. 1 by displaying their positions $u_{1}$ and $v_{1}$ relative to $\mathrm{L} 1 \mathrm{a}$, where these two quantities and focal length $f_{1}$ are related by $u_{1}{ }^{-1}+v_{1}{ }^{-1}=f_{1}{ }^{-1}$. Since the focus of L2a and the sample plane are conjugate, the illuminated spot is circular and proportional to the impinging beam cross section at the diffraction element. The size of the illumination spot can therefore be controlled by expanding the spot size on the diffractive element itself. This differs from the way the illumination-spot size is controlled in the case of Stout and Axelrod in which a diffusive element is used.

An alternative way of implementing ODFSM is depicted in Fig. 2. This setup is based on the original arrangement of Stout and Axelrod. However, 


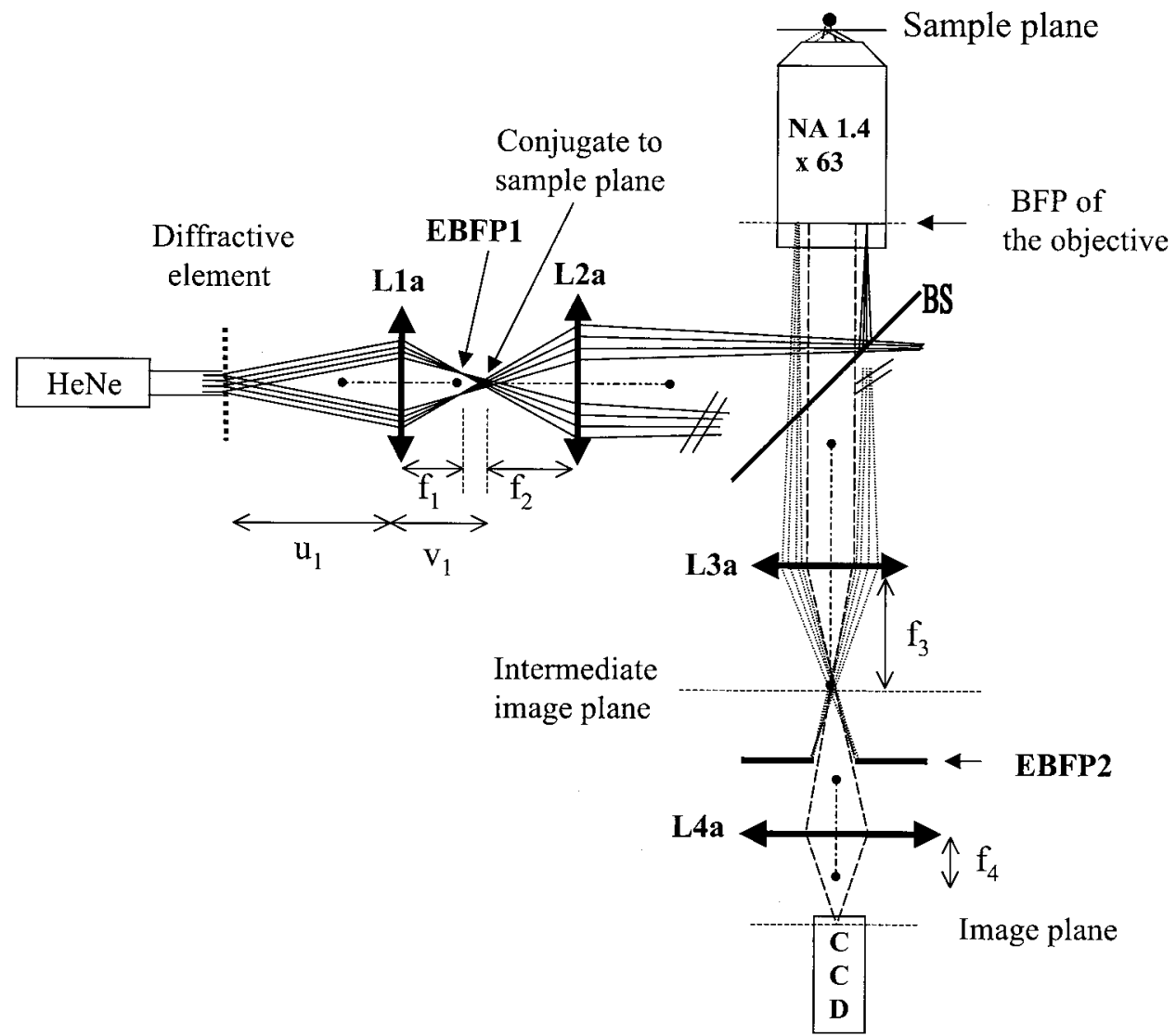

Fig. 1. Configuration for implementation of ODFSM: the diffraction element method: solid lines, the laser beam passing through a diffractive element that concentrates $80 \%$ of the beam into a diverging ring. Lenses L1a, L2a, and the $50 \%$ transmission neutral density beam splitter, BS, steer the beam into a Zeiss NA 1.4, 63× objective to form nearly evanescent illumination. Dashed lines, the illumination beam reflected by the coverslip-water interface back into the objective. Lens L3a forms an image of the backreflected beam on the annular block placed at the equivalent back focal plane, EBFP2, thereby filtering out the nonscattered light. L4a images the scattered light from the beads unto the CCD device. For clarity, incoming rays impinging on the BFP from only one side of the objective and their associated reflected outcoming rays are shown passing through the objective. With this method the illumination area in the sample is a spot with a size of $\sim 30 \mu \mathrm{m}$ in our setup. The pair of focal spots on both sides of each lens (of focal distance $f_{i}$ ) are shown as full circles and have been joined by dotted-dashed lines.

instead of a full annulus, we illuminated the BFP of the objective with a beam of crescent-shaped cross section, created by blocking a laser beam at the EBFP with an opaque, slightly off-axis disk. The beam entered the objective from one side, while the reflected rays emerged from the diametrically opposed side, where they were blocked by an opaque screen covering less than half of the objective's back aperture. Although the block's position does not coincide with the BFP, complete blocking of the reflected beam was achieved. No appreciable reduction in resolution was observed owing to the presence of the block. Therefore light scattered by particles in the sample exited from the same side of the objective as the rays entering for illumination, achieving the same effect as in Fig. 1. A diffusing screen between lenses L1b and L2b, at a plane conjugate to the sample plane, converted the laser beam into an extended source, thereby illuminating a wide area on the sample. Lens L4b was finally used to form an image of the sample plane onto the CCD. This configuration also can be used for spotlike illumination of the sample by removal of the diffusing screen. ${ }^{9}$

We emphasize that these two methods are different in many aspects regarding the positioning of the lenses. However, the net result is the same, namely, only part of the BFP is illuminated and the reflected illumination beam is blocked at the EBFP. Furthermore we point out that one can get a dark-field effect without conjugating EBFP1 and EBFP2 with BFP. However, it is beneficial to do so: At the BFP there is a field stop inherent in the system, because the NA of the objective is limited. In the illumination part of the ODFSM scheme, a field stop that is not located at the EBFP would result in further blocking of illumination rays and therefore to a loss of illuminating intensity. At the collection path a field stop not located at the EBFP would block more scattered rays coming from the sample plane compared with the same block at the EBFP. Hence conjugating EBFP1 and EBFP2 with BFP yields the best conditions.

Both of the configurations shown in Figs. 1 and 2 


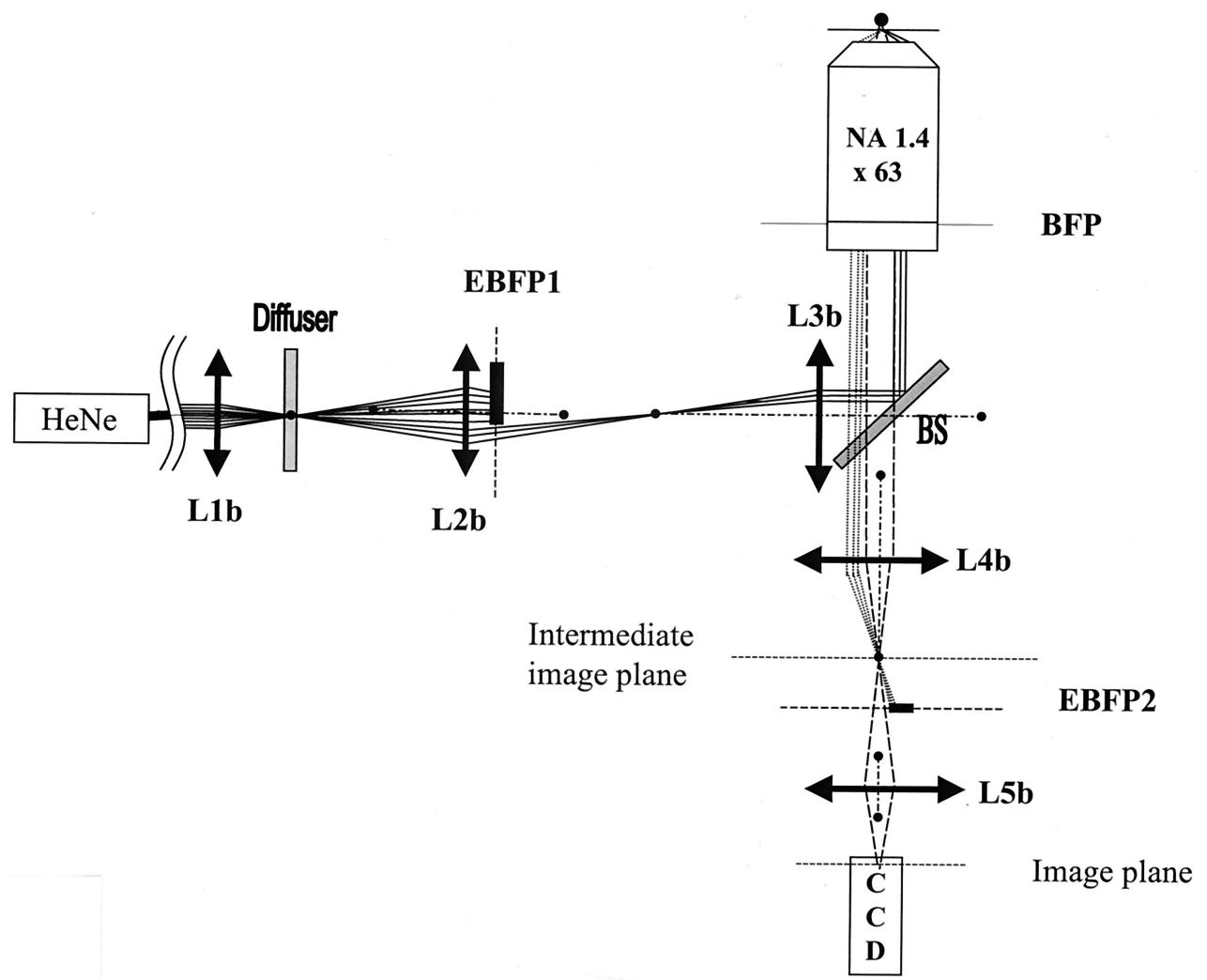

Fig. 2. Configuration for implementation of ODFSM: The blocking disk method. This method can produce either spotlike illumination in which a focused beam is conjugated to the image plane or large-field illumination. In the latter case a rotating diffusing screen is placed at a plane conjugate to the sample plane at the focus of lens L1b. The screen converts the laser beam into an extended source. A large block placed at the equivalent back focal plane, EBFP1, blocks everything aside for a small crescent-shaped cross section. The arc is incident on one edge of the back aperture of the objective, and the backreflected light is blocked on the diametrically opposed side at an equivalent back focal plane, EBFP2, created by lens L4b. The scattered light is imaged by lens L5b on the CCD.

were implemented around a home-built inverted microscope with a Zeiss $63 \times$ objective with a nominal NA of 1.4. For illumination, light from a $10-\mathrm{mW}$ $\mathrm{He}-\mathrm{Ne}$ laser at $633 \mathrm{~nm}$ or a $30-\mathrm{mW}$ argon laser at 488 nm was used, but these sources can be substituted by other well-collimated monochromatic noncoherent sources. Images were captured with a CCD camera (Cohu 4710) and recorded on video for further analysis.

ODFSM conditions can also be achieved with a commercial epi-illumination microscope. This can be done, for example, on a Zeiss Axiovert 135 microscope by following the procedures outlined by Stout and Axelrod for the illumination path and by choosing the front port for the detection path. To find an appropriate equivalent BFP to place the field stop and block reflected rays on the detection path, it is necessary to add lens L2c as illustrated in Fig. 3. L2c forms a new equivalent back focal plane, EBFP*, outside the microscope body, conjugate to the EBFP formed by the lenses inside the microscope body. In a Zeiss Axiovert 135 microscope, there are three lenses after the objective. The first one images the sample in its focus and further down on the EBFP. The other two lenses are located between the sample image and the EBFP. These lenses form a new image of the sample outside the microscope body. We denote the last lens as L1c. L2c was placed just at the outport between the EBFP and the image formed by L1c. To find the EBFP* after L2c, we illuminated the objective with parallel illumination by introducing a pinhole in the BFP of the condenser and adjusted the objective into its working position by focusing on a sample by using the eyepiece. Then we looked for the plane where light emerging from the front port was focused by L2c into a point. The field stop was placed at this position, which is the EBFP*. Finally, another lens, L3c, was placed beyond $\mathrm{EBFP}^{*}$ to form an image for detection with a CCD camera. The image plane was determined by matching the features observed beyond L3c to those that were observed by the eyepiece simultaneously. For our Zeiss Axiovert 135, we used two lenses with a focal length of $50 \mathrm{~mm}$ for L2c and L3c. The distance between L2c and the block was $100 \mathrm{~mm}$. The overall length between the microscope outport and the CCD chip was $225 \mathrm{~mm}$.

Experiments in which we followed the Brownian motion of beads tethered to glass slides by DNA molecules of different length were carried out at room temperature in a buffer consisting of $10-\mathrm{mM}$ Tris $\mathrm{Cl}$, 0.1-mM ethylene diamine tetra-acetic acid (EDTA), 200-mM KCl, 5\% dimethyl sulfoxide (DMSO), $0.2-\mathrm{mM} \mathrm{DTT}$, and $\alpha$-casein at $0.2 \mathrm{mg} / \mathrm{ml}$ to keep the 


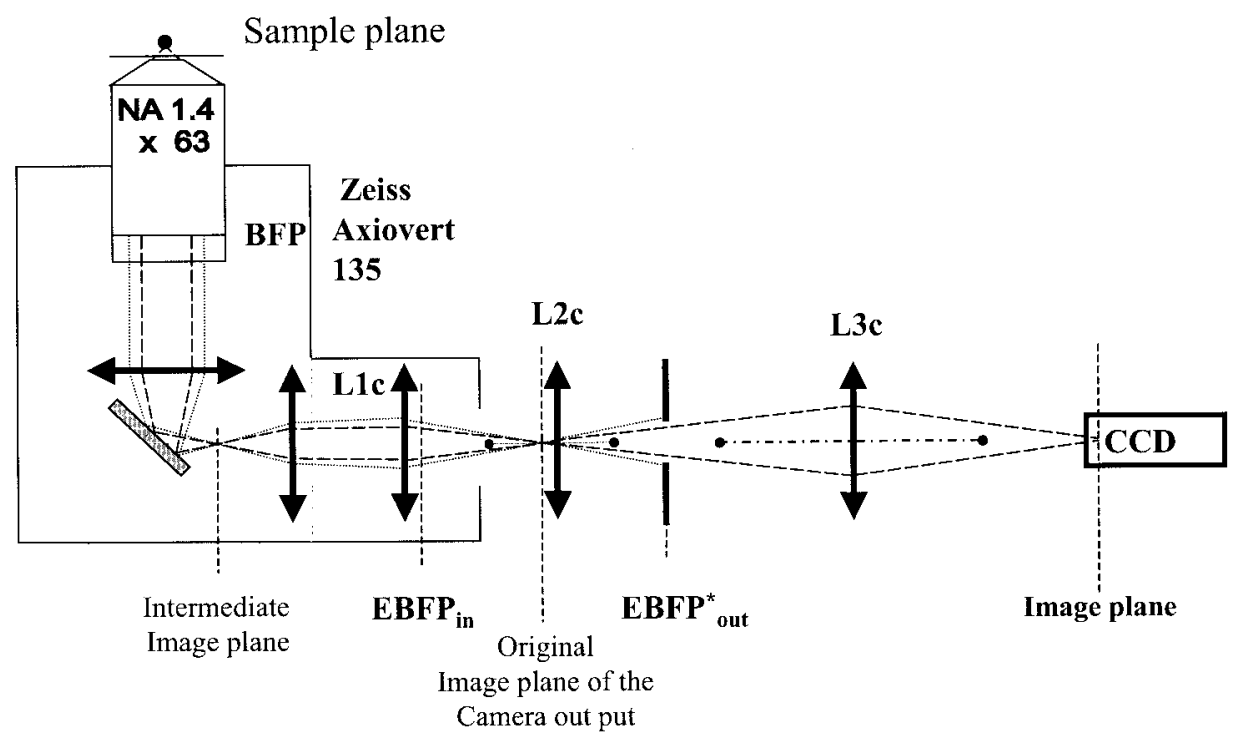

Fig. 3. Modifications in the detection path for the implementation of ODFSM in a Zeiss Axiovert 135 microscope. Lenses L2c and L3c and the beam block are added between the microscope's outport and a CCD camera.

DNA molecules or beads from sticking to the glass surface. The lengths of the molecules were 891, 1073, 1199, 1298, 1433, and 1495 base pairs. (A single base pair corresponds to $0.34 \mathrm{~nm}$.) To stick the DNA molecules to both the glass slide and beads, we followed published procedures. ${ }^{7}$

\section{Results}

We show in Fig. 4 typical images of three beads with diameters of 290,490 , and $810 \mathrm{~nm}$ stuck to the glass surface, obtained both with ordinary bright-field illumination and ODFSM, using the configuration in Fig. 1. Each image comprises a single video frame. The

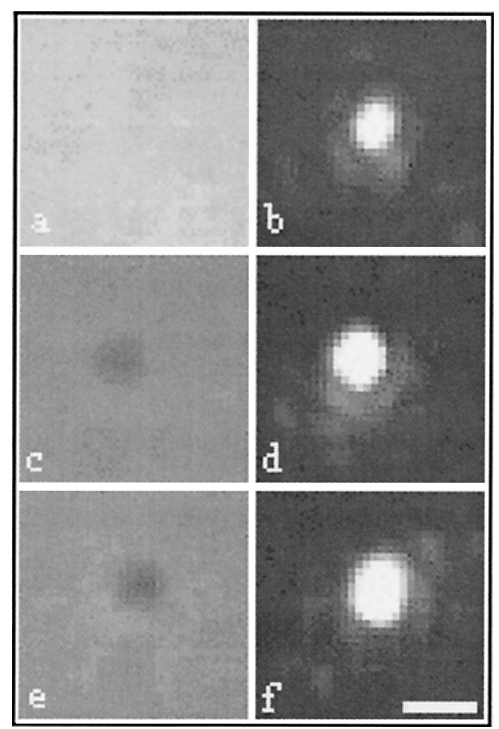

Fig. 4. Images of beads of diameters of 290,490 , and $810 \mathrm{~nm}$ (top to bottom) with, left, bright-field illumination and, right, ODFSM. The scale bar corresponds to $1 \mu \mathrm{m}$. 290-nm bead is hardly visible with bright-field illumination, whereas it can be visualized clearly with ODFSM. Removing the block at the EBFP2 washes out the images of the beads, preventing their detection.

Figure 5 shows a series of images of beads of different sizes stuck to the glass surface, ranging in diameter from 60 to $810 \mathrm{~nm}$. No contrast enhancement or average has been performed on any of these images, except for the autogain of the camera. We measured the signal-to-noise ratio $(\mathrm{S} / \mathrm{N})$ in these images by taking the ratio of the average intensity of the bead image $I_{\text {bead }}$ minus the average intensity of the

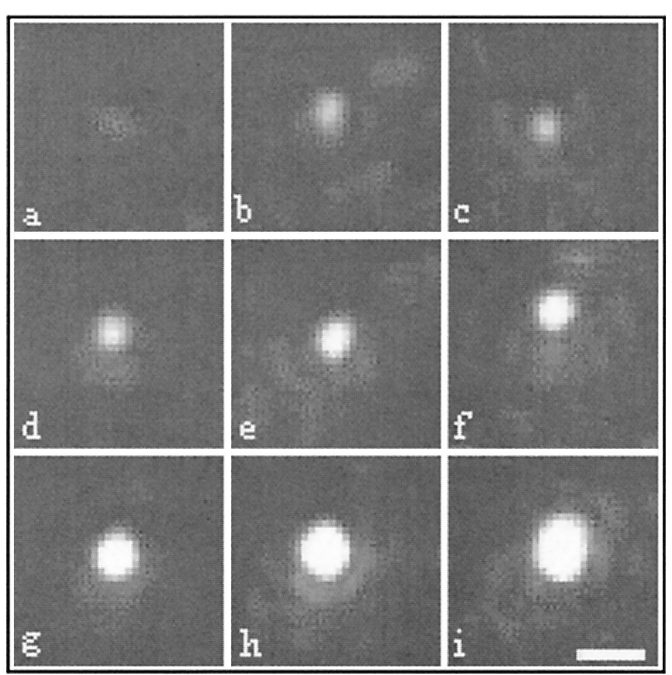

Fig. 5. Images of beads of different sizes obtained with ODFSM with the configuration shown in Fig. 1: a, $60 \mathrm{~nm}$; b, $72 \mathrm{~nm}$; c, 86 $\mathrm{nm}$; d, $110 \mathrm{~nm}$; e, $160 \mathrm{~nm}$; f, $200 \mathrm{~nm}$; g, $290 \mathrm{~nm}$; h, $490 \mathrm{~nm}$; i 810 $\mathrm{nm}$. The scale bar corresponds to $1 \mu \mathrm{m}$. 


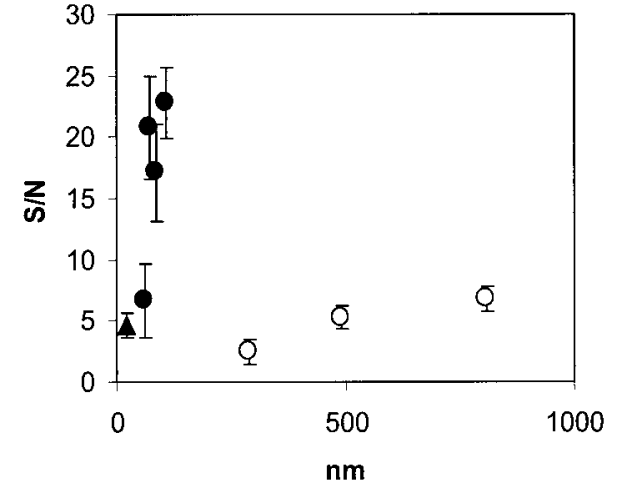

Fig. 6. Signal-to-noise ratio as a function of the polystyrene bead diameter by using, $\bigcirc$, bright-field illumination; $\bullet$, ODFSM; $\boldsymbol{\Delta}, \mathrm{S} / \mathrm{N}$ for a gold particle of $20 \mathrm{~nm}$. For polystyrene beads observed with ODFSM, S/N has been calculated only for diameters below $110 \mathrm{~nm}$ for which the images do not show saturation. These data correspond to the images shown in Figs. 4 and 5. Note that beads with a diameter of $60 \mathrm{~nm}$ are observed with a value of $\mathrm{S} / \mathrm{N} \sim 6$.

background $I_{\text {background }}$ divided by the standard deviation of the background $\sigma_{\text {background }}$ :

$$
\frac{S}{N}=\frac{I_{\text {bead }}-I_{\text {background }}}{\sigma_{\text {background }}} .
$$

This definition of $\mathrm{S} / \mathrm{N}$ is robust with respect to linear transformations such as autogain in the camera. Figure 6 shows the measured $\mathrm{S} / \mathrm{N}$ as a function of the bead diameter both for bright-field illumination and for ODFSM. Clearly, the S/N with ODFSM is higher than with bright-field illumination. Furthermore the graph shows that with ODFSM it is possible to detect beads below $300 \mathrm{~nm}$ with high values of $\mathrm{S} / \mathrm{N}$. In contrast, the same beads are not detectable by using bright-field illumination, precluding measurement of S/N. In the dark-field mode the beads were so bright compared with the background that images of the beads whose size is above $110 \mathrm{~nm}$ were saturated. For this reason the value of their $\mathrm{S} / \mathrm{N}$ was not calculated. Nonetheless we estimate a lower bound of $\mathrm{S} / \mathrm{N}>25$. In comparison the $\mathrm{S} / \mathrm{N}$ for bright-field visualization did not exceed 6 , even for 800-nm beads.

We were also able to detect small 20-nm gold particles stuck to a glass surface by using the setup described in Fig. 1 and an argon laser for illumination. The image (not shown) appears as a bright spot with a $\mathrm{S} / \mathrm{N}$ of 5 . We were not able to observe gold beads in motion, even when we used a solution that contained an equal amount of glycerol and water by volume to increase viscosity. We believe this to be due to the large diffusion coefficient of the beads that prevented them from staying localized during the integration time of the camera. We now illustrate the use of ODFSM by studying the Brownian motion of $290-\mathrm{nm}$ beads tethered to a glass slide by single DNA molecules of different length. This experiment was carried out by using the configuration of Fig. 1, and illumination was provided by an argon laser $(488 \mathrm{~nm})$ with a beam intensity of $30 \mathrm{~mW}$.

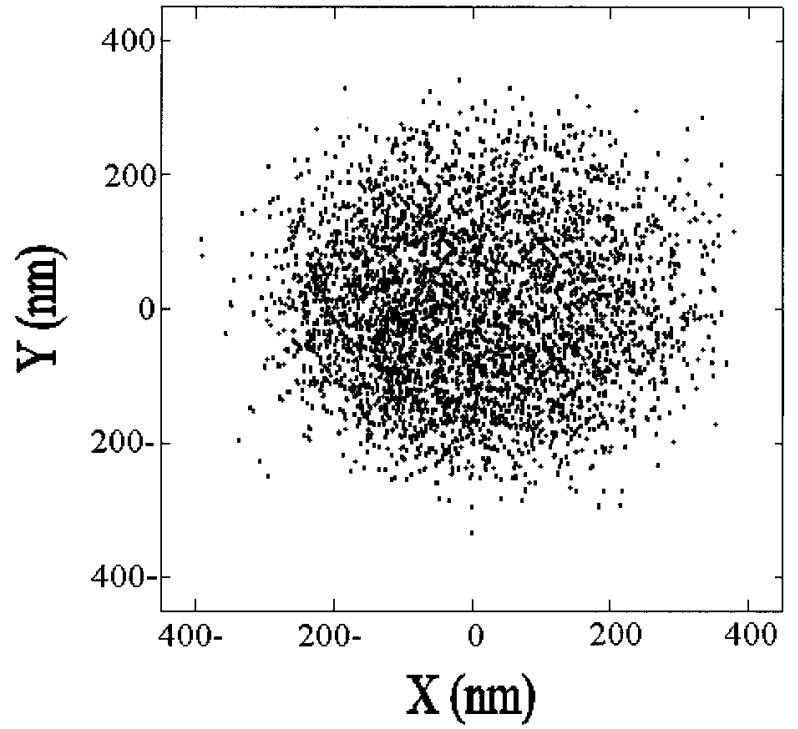

Fig. 7. The $x y$ position of the center of a bead tethered to a glass slide by a short DNA molecule (1073 base pairs) at different times. Data were accumulated for $400 \mathrm{~s}$.

The motion of the beads was recorded in video for $20 \mathrm{~min}$ at a rate of $10 \mathrm{~Hz}$. To record continuously for that period, it proved important to stabilize the temperature of the cell and the objective within $5 \mathrm{mK}$. We estimated the amplitude of their Brownian motion $A_{B M}$ as the width at half-maximum of radial density distribution functions $f(r)$ extracted from the recordings. The analysis was done with IDL routines. ${ }^{13}$ A Gaussian mask was applied to each image in a recording for contrast enhancement, and then the $x$ and $y$ coordinates of the center of mass of the resulting object were estimated. After subtracting from each coordinate its average throughout the run, we plotted the $x$ and $y$ coordinates against each other as shown in Fig. 7. Next the number of events at a given distance $r$ from the center of mass was counted, and this value was divided by $r$ to obtain $f(r)$. The result is shown in Fig. 8. The width of this distribution function at half-height is a measure of the amplitude of the Brownian motion $A_{B M}$. The

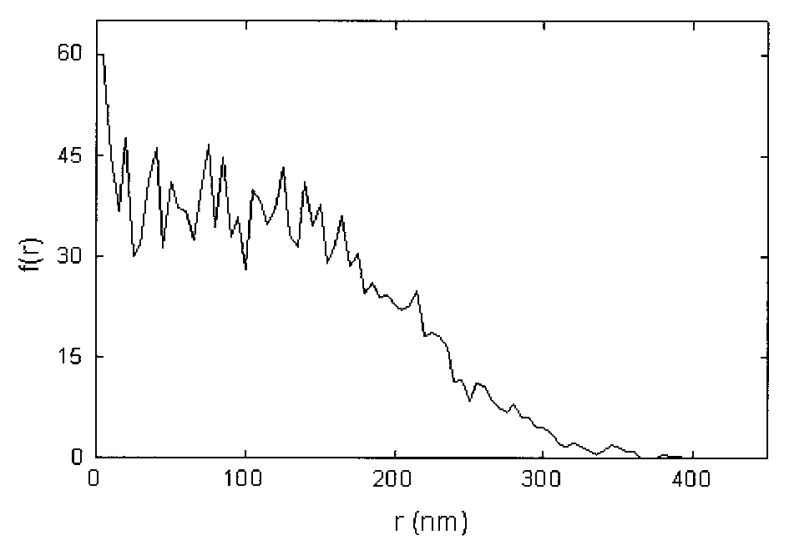

Fig. 8. Radial distribution function $f(r)$ corresponding to the data shown in Fig. 7. 


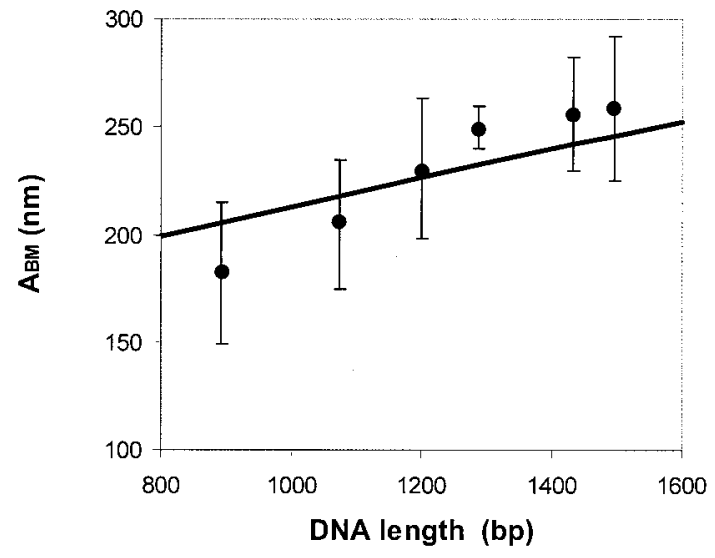

Fig. 9. Measured amplitude of Brownian motion $A_{B M}$ of 290-nm beads tethered to a glass surface by DNA molecules as a function of, $\bullet$ DNA length. The solid line denotes the calculated amplitude of Brownian motion $A_{B M}$ as described in the text.

dependence of $A_{B M}$ on tether length $L$ is shown in Fig. 9. Each experimental data point comprises independent measurements on at least five different beads. The error bars that represent one standard deviation of the data reflect differences in the microenvironment of each molecule. To interpret these data, we follow the work of Qian and Elson ${ }^{14}$ who regarded a bead connected to a DNA tether as an extra segment of the tether, albeit of a different segment size. In their model the average end-to-end distance of the DNA tether $D$ and the bead radius $\rho$ add up in quadratures:

$$
A_{B M}=\left(\rho^{2}+D^{2}\right)^{1 / 2}
$$

This equation is valid only if the bead is free to fluctuate. If the tether is too short, the proximity of the bead to the surface will prevent the bead from fluctuating. In our case $\rho$ and $D$ are comparable, when we approximate $D$ as the average radius of gyration $R_{F}$ of a free molecule in the solution by using the equation ${ }^{14} R_{F}=a N^{0.6}$. Here $a$ is the segment length and $N$ is the number of segments. We take $a$ to be the persistence length whose value we assume to be $50 \mathrm{~nm}$ (Ref. 4) and $N$ to be the number of persistence lengths in the chain. In Fig. 9 we compare these calculations with the experimental values of $A_{B M}$. There is good agreement between our measurements and the model. Nonetheless we point out that the DNA molecules in our experiments cannot fluctuate freely, because they are constrained by being attached to both the surface and the bead. Thus this model represents only a first approximation to the experimental situation.

Yin et al. ${ }^{15}$ performed similar experiments using DIC to measure the amplitude of Brownian motion. Their definition of this quantity is, however, different from ours. Nonetheless their results and ours are in agreement within the framework of Qian and Elson's model. ${ }^{14}$

\section{Discussion}

The key idea in ODFSM is to block the rays reflected by the water-glass interface in a microscope in which objective-type annular illumination is implemented. In general, dark-field illumination methods block the zeroth order of the illumination by introducing an opaque disk. This prevents direct transmission of light not scattered in the sample, thereby enabling the observation of phase objects ${ }^{16}$ and enhancing contrast, thus increasing the $\mathrm{S} / \mathrm{N}$ ratio.

The use of ODFSM to detect particles in the proximity of an interface offers several advantages over other detection schemes. First, objects can be viewed and tracked continuously and indefinitely. Long times are hard to achieve with other techniques such as fluorescence, where photobleaching places a practical limit of an hour or less for detection. Second, the dark-field illumination inherent in ODFSM allows for detection of scattered light from very small particles $(<100 \mathrm{~nm})$, unlike other scattering schemes designed for large particles. Third, using ODFSM with completely evanescent illumination may allow for three-dimensional tracking by use of the sensitive exponential drop in intensity as a function of height, which is characteristic of total internal reflection techniques. We point out that purely evanescent illumination is hard to achieve with objectives with a nominal NA of 1.4, since in practice the latter is in fact slightly smaller (new objectives with a higher NA are becoming available). Furthermore the presence of macromolecules and other solutes may increase slightly the index of refraction of the sample, restricting further the condition for total internal reflection. To satisfy the condition of purely evanescent illumination, the beam at the objective's back aperture must be very thin and is therefore hard to collimate precisely with the objective rim.

In applications such as ours, where only the transverse bead displacement is monitored, a small portion of nonevanescent light can be tolerated, with the additional advantage that objects that are farther out of the surface can be detected. ODFSM can be used in a variety of applications, in particular where single-molecule techniques are called for. For example, ODFSM has been used to study the compaction of short single DNA molecules tethered to a surface by the integration host factor, a histonelike protein that plays a fundamental role in shaping the genetic material in E. coli. ${ }^{17}$ The use of dark-field methods to track small beads has been demonstrated recently in a beautiful study of single kinesin molecules bound to 200-nm gold particles, moving along microtubules. ${ }^{18}$ Displacements below $8 \mathrm{~nm}$ were measured with a bandwidth of $20 \mathrm{kHz}$. This implementation of the dark-field effect involved two microscope objectives. One could use ODFSM for similar purposes by using only one high numerical objective for both illumination and detection.

The idea of using oblique illumination has been implemented in the past to enhance image contrast and observe phase objects without using special- 
purpose objectives. ${ }^{10,11}$ In ODFSM we create an additional EBFP far away from the objective, which gives us the flexibility to block unwanted light as desired. Furthermore, by illuminating through the objective, which then also plays the role of a condenser, we make use of its high numerical aperture and benefit from all the advantages of objective-type illumination. ${ }^{9}$ These features constitute improvement over other schemes proposed in the past. ${ }^{10,11}$

ODFSM can be implemented easily in most epiillumination microscopes after simple modifications. It provides a good alternative to other schemes for tracking small particles such as differential interference contrast as well as other light-scattering methods. ${ }^{19-21}$

We acknowledge useful conversations with T. Kam, N. Davidson, M. Elbaum, and D. Bensimon and help from Y. Schopen.

\section{References}

1. D. C. Prieve and N. A. Frej, "Total internal reflection microscopy: a quantitative tool for the measurement of colloidal forces," Langmuir 6, 396-403 (1990).

2. T. R. Strick, J-F. Allemand, A. Bensimon, D. Bensimon, and V. Croquette, "The elasticity of a single supercoiled DNA molecule," Science 271, 1835-1837 (1996).

3. G. V. Shivashankar, M. Feingold, O. Krichevsky, and A. Libchaber, "RecA polymerization on double-stranded DNA by using single-molecule manipulation: the role of ATP hydrolysis," Proc. Natl. Acad. Sci. USA 96, 7916-7921 (1999).

4. T. R. Strick T. V. Croquette, and D. Bensimon, "Singlemolecule analysis of DNA uncoiling by a type II topoisomerase," Nature (London) 404, 901-904 (2000).

5. H. Yin, M. D. Wang, K. Svoboda, R. Landick, S. M. Block, and J. Gelles, "Transcription against an applied force," Science 270, 1653-1657 (1995).

6. G. Zocchi, "Proteins unfold in steps," Proc. Natl. Acad. Sci. USA 94, 10647-10651 (1997).

7. L. Finzi and J. Gelles, "Measurement of lactose repressormediated loop formation and breakdown in single DNA molecules," Science 267, 378-380 (1995).
8. H. C. van de Hulst, Light Scattering by Small Particles (Dover, New York, 1981).

9. A. L. Stout and D. Axelrod, "Evanescent field excitation of fluorescence by epi-illumination microscopy," Appl. Opt. 28, 5237-5242 (1989).

10. D. Axelrod, "Zero-cost modification of bright-field microscopes for imaging phase gradient on cells: Schlieren optics," Cell Biophys. 3, 167-173 (1981).

11. W. B. Piekos, "Diffracted-light contrast enhancement: a reexamination of oblique illumination," Microsc. Res. Tech. 46, 334-337 (1999), and references therein.

12. R. Ozeri, L. Khaykovich, N. Friedman, and N. Davidson, "Large volume single-beam dark optical trap for atoms using binary phase elements," J. Opt. Soc. Am. B 17, 1113-1116 (2000).

13. J. C. Crocker and D. G. Grier, "Methods of digital video microscopy for colloidal studies," J. Colloid Interface Sci. 179, 298-310 (1996).

14. H. Qian and E. L. Elson, "Quantitative study of polymer conformation and dynamics by single-particle tracking," Biophys. J. 76, 1598-1605 (1999).

15. H. Yin, R. Landick, and J. Gelles, "Tethered particle motion method for studying transcript elongation by a single RNA polymerase molecule," Biophys. J. 67, 2468-2478 (1994).

16. S. G. Lipson and H. Lipson, Optical Physics (Cambridge University, Cambridge, UK, 1995).

17. B. M. J. Ali, R. Amit, I. Braslavsky, A. B. Oppenheim, O. Gileadi, and J. Stavans, "Compaction of single DNA molecules induced by binding of integration host factor (IHF)," Proc. Natl. Acad. Sci. USA 98, 10658-10663 (2001).

18. M. Nishiyama, E. Muto, Y. Inoue, T. Yanagida, and H. Higuchi, "Substeps within the 8-nm step of the ATPase cycle of single kinesin molecules," Nature Cell Biol. 3, 425-428 (2001).

19. R. Bar-Ziv, A. Meller, T. Tlusty, E. Moses, J. Stavans, and S. A. Safran, "Localized dynamic light scattering: probing single particle dynamics at the nanoscale," Phys. Rev. Lett. 78, 154157 (1997).

20. A. Meller, R. Bar-Ziv, T. Tlusty, E. Moses, J. Stavans, and S. A. Safran, "Localized dynamic light scattering: a new approach to dynamic measurements in optical microscopy," Biophys. J. 74, 1541-1548 (1998).

21. G. V. Shivashankar, G. Stolovitzky, and A. Libchaber, "Backscattering from a tethered bead as a probe of DNA flexibility," Appl. Phys. Lett. 73, 291-293 (1998). 